\title{
Pretransplant transcriptomic signature in peripheral blood predicts early acute rejection
}

\author{
Weijia Zhang, ${ }^{1}$ Zhengzi Yi, ${ }^{1}$ Chengguo Wei, ${ }^{1}$ Karen L. Keung, ${ }^{2}$ Zeguo Sun, ${ }^{1}$ Caixia Xi, ${ }^{1}$ \\ Christopher Woytovich, ${ }^{1}$ Samira Farouk, ${ }^{1}$ Lorenzo Gallon, ${ }^{3}$ Madhav C. Menon, ${ }^{1}$ Ciara Magee, ${ }^{4}$ \\ Nader Najafian, ${ }^{4}$ Milagros D. Samaniego, ${ }^{5}$ Arjang Djamali, ${ }^{6}$ Stephen I. Alexander, ${ }^{2}$ Ivy A. Rosales, \\ Rex Neal Smith, ${ }^{7}$ Philip J. O'Connell, ${ }^{2}$ Robert Colvin, ${ }^{7}$ Paolo Cravedi,, and Barbara Murphy ${ }^{1}$ \\ 'Division of Nephrology, Department of Medicine, Icahn School of Medicine at Mount Sinai, New York, New York, USA. \\ ${ }^{2}$ Department of Medicine, Westmead Clinical School, The University of Sydney, Sydney, Australia. ${ }^{3}$ Department of \\ Medicine-Nephrology and Surgery-Organ Transplantation, Northwestern University Feinberg School of Medicine, Chicago, \\ Illinois, USA. ${ }^{4}$ Department of Medicine, Brigham and Women's Hospital, Boston, Massachusetts, USA. \\ ${ }^{5}$ Henry Ford Hospital, Detroit, Michigan, USA. 'Division of Nephrology, Department of Medicine, University of Wisconsin, \\ Madison, Wisconsin, USA. 'Department of Pathology, Massachusetts General Hospital, Harvard Medical School, \\ Boston, Massachusetts, USA
}

Conflict of interest: BM reports stock in RenalytixAI. BM and WZ report patents (a) US Provisional Patent Application F\&R reference 27527-0134P01, serial number 61/951,651, filed March 2014: Method for identifying kidney allograft recipients at risk for chronic injury; (b) US Provisional Patent Application: Methods for Diagnosing Risk of Renal Allograft Fibrosis and Rejection (miRNA); (c) US Provisional Patent Application: Method For Diagnosing Subclinical Acute Rejection by RNA sequencing Analysis of A Predictive Gene Set; and (d) US Provisional Patent Application: Pretransplant prediction of after transplant acute rejection. JPO is a consultant for CSL Behring and Vitaeris.

Copyright: () 2019 American Society for Clinical Investigation

Submitted: January 19, 2019

Accepted: April 23, 2019

Published: June 6, 2019.

Reference information: /CI Insight. 2019;4(11):e127543. https://doi. org/10.1172/jci.insight.127543.
Commonly available clinical parameters fail to predict early acute cellular rejection (EAR, occurring within 6 months after transplant), a major risk factor for graft loss after kidney transplantation. We performed whole-blood RNA sequencing at the time of transplant in 235 kidney transplant recipients enrolled in a prospective cohort study (Genomics of Chronic Allograft Rejection [GoCAR]) and evaluated the relationship of pretransplant transcriptomic profiles with EAR. EAR was associated with downregulation of NK and CD8 ${ }^{+} \mathrm{T}$ cell gene signatures in pretransplant blood. We identified a 23-gene set that predicted EAR in the discovery $(n=81$, and $A U C=0.80$ ) and validation $(n=74$, and $A U C=0.74)$ sets. Exclusion of recipients with 5 or 6 HLA donor mismatches increased the AUC to 0.89 . The risk score derived from the gene set was also significantly associated with acute cellular rejection after 6 months, antibody-mediated rejection and/or de novo donor-specific antibodies, and graft loss in a cohort of 154 patients, combining the validation set and additional GoCAR patients with surveillance biopsies between 6 and 24 months $(n=80)$ posttransplant. This 23 -gene set is a potentially important new tool for determination of the recipient's immunological risk before kidney transplantation, and facilitation of an individualized approach to immunosuppressive therapy.

\section{Introduction}

Kidney transplantation is the treatment of choice for patients with end-stage renal disease (1). However, despite remarkable improvements in 1-year graft survival over the last decades, in each subsequent year after transplant, approximately 3\% of kidney allograft recipients return to dialysis or require retransplantation, making the rates of late graft failure relatively unchanged since the 1990s (2, 3).

Chronic allograft damage, or interstitial fibrosis and tubular atrophy of unknown cause, account for most of the cases of graft loss (4-6). This has caused an increase in genomic research aimed at understanding and contrasting the mechanisms responsible for these late events and identifying early biomarkers for prediction of fibrosis (7). Though mechanisms responsible for late development of alloantibodies and graft fibrosis are still largely unknown, experimental and clinical evidence has been accumulating showing that early alloreactive responses have a major impact on long-term graft outcomes $(8,9)$. EAR, both clinically manifested and subclinical $(10)$, is a common event and negatively affects long-term graft survival in patients receiving standard immunosuppressive regimens (11). Therefore, preventing EAR is crucial to improve long-term graft outcomes.

One of the major issues with current immunosuppressive protocols is they are not tailored to the individual patients' need $(12,13)$. In clinical practice, immunosuppressive therapy is often decided based on broad clinical criteria, including anti-HLA antibodies, ethnicity, prior transplantations, and recipient age. 
However, these indicators perform poorly in predicting individual risk for development of acute cellular rejection (AR). Because a result, most patients receive a standardized immunosuppressive protocol, resulting in some individuals being exposed to insufficient or excessive immunosuppression, leading to AR or complications associated with overimmunosuppression, respectively $(12,13)$. Early identification of individuals at highest risk of AR could allow targeted therapies aimed at improving long-term outcomes.

Evidence exists that the phenotype (14) and function (15) of the immune system in patients before renal transplantation affect the risk for subsequent AR after transplantation, but no biomarker has been identified to quantify this risk. Herein, we tested the hypothesis that transcriptome profiling of the blood of patients before renal transplant predicts AR episodes and graft survival.

\section{Results}

Study population. The study included 235 kidney transplant recipients who were followed for an average $\pm \mathrm{SD}$ of $58.14 \pm 19.50$ months (Figure 1). Patients were mainly white (159 of 235, 67.7\%) and received deceased (117 of $235,49.8 \%$ ) and living (118 of 235, 50.2\%) grafts. Overall, pretransplant donor and recipient characteristics were similar among EAR D $(n=81)$ and V $(n=74)$ cohorts with serial surveillance biopsies between 1 and 24 months after transplant and a cohort with late surveillance biopsies between 6 and 24 months after transplant (Late/L, $n=80$ ) (Table 1).

EAR is associated with LAR and graft loss but is not predicted by baseline clinical characteristics. Within EAR D and V cohorts $(n=155), 58$ patients $(37.4 \%)$ developed EAR (borderline, $n=43$; $1 \mathrm{~A}$ or above, $n=15$ ) (Supplemental Table 1; supplemental material available online with this article; https://doi.org/10.1172/jci. insight.127543DS1). Nine out of 58 EAR cases were associated with signs of ABMR, whereas 4 patients had isolated ABMR. Six EAR and 6 non-EAR patients developed de novo DSAs. Patients with EAR had a higher chance of developing LAR ( $>6$ months after transplant) than those who did not have EAR (57.89\% vs. $30.43 \% ; P=0.005$; Supplemental Figure $1 \mathrm{~A}$ ). EAR was also significantly associated with accelerated graft loss (time to graft loss \pm SD: $19.8 \pm 15.8$ vs. $40.9 \pm 23.3$ months in patients with or without EAR, respectively; log-rank $P=0.014$ ) (Supplemental Figure $1 \mathrm{~B}$ ). Both subclinical borderline and at least $1 \mathrm{~A}$ EAR episodes were similarly associated with graft loss (not shown).

Next, we examined the correlation between pretransplant demographic and clinical characteristics and EAR. Univariate analysis showed kidney disease, recipient age, presence of anti-HLA antibodies, and induction therapy to be independently associated with EAR at $P<0.05$. In multivariate analysis, kidney disease, recipient age, and presence of pretransplant anti-HLA antibodies remained significantly $(P<0.05)$ or marginally significantly $(P<0.1)$ associated with development of EAR (Supplemental Table 2), but these 3 factors did not predict EAR either individually or in combination (AUC $=0.59$ with $95 \%$ CI: 0.49-0.69; Supplemental Figure 1C).

Patients with EAR have a distinct transcriptomic profile in pretransplant peripheral blood. RNA sequencing of pretransplant peripheral blood of kidney transplant recipients was analyzed using the approach described in the Supplemental materials and depicted in Supplemental Figure 2. Differential analysis in the D cohort revealed 688 upregulated and 653 downregulated genes at a significant $P$ value, less than 0.05 , in patients with $\operatorname{EAR}(n=32)$ compared with those without EAR $(n=49)$ (Figure 2A). Canonical pathway and Gene Ontology enrichment analysis indicated that upregulated genes were involved in membrane transport and cytokine production/immunity, whereas downregulated genes were enriched for functions/pathways of IL-12 signaling and NK cell- and T cell-mediated cell toxicity, which was confirmed by Ingenuity Pathway Analysis and Gene Set Enrichment Analysis (Figure 2B, Supplemental Figures 3 and 4, and ref. 16). In keeping with this, the pretransplant protein levels of serum IL-12 in patients with EAR was lower compared with patients without EAR (Figure 2C). Expression correlation network analysis for DEGs further identified core clusters in NK cell and $\mathrm{CD}^{+} \mathrm{T}$ cell cytotoxicity as highly correlative (Figure 2D). Similarly, immune cell enrichment analysis using the ImmGen database (17) showed downregulated genes were enriched for NK or $\mathrm{CD}^{+} \mathrm{T}$ cells but upregulated for neutrophils and monocytes (Figure 2E and Supplemental Figure 5A). This was also consistent with additional analysis showing decreased NK and CD8 ${ }^{+}$ $\mathrm{T}$ cell populations, which were deconvoluted from the bulk blood RNA sequencing data using expression profiles of sorted human immune cells (Supplemental Figure 5B and ref. 18).

Pretransplant peripheral blood transcriptomic signature predicts EAR. To identify the gene set in the pretransplant blood that optimally predicts EAR, we first identified a focus gene set of 70 genes from DEGs associated with EAR using criteria described in Supplemental Methods (Supplemental Table 3). Using a permutation-based approach and by determining the cumulative gene risk score (see Supplemental materials), we narrowed this 


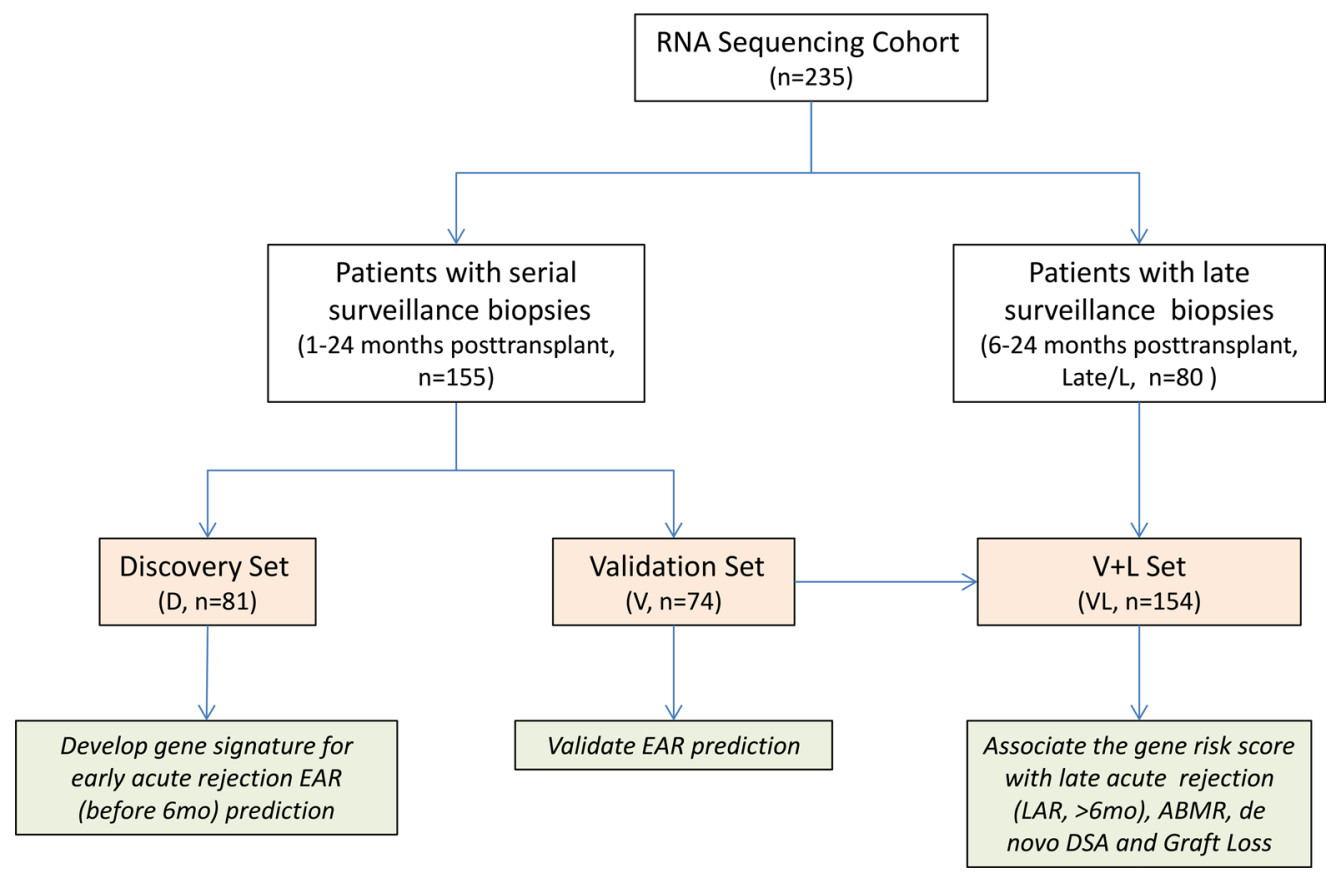

Figure 1. Description of study cohorts. The study included 235 GoCAR kidney transplant recipients with good-quality pretransplant blood RNA samples. Of the 235,155 had pretransplant biopsies and serial after transplant surveillance biopsies until 24 months after transplant per protocol, whereas 80 had only late surveillance biopsies only between 6 and 24 months after transplant. Out of 155 patients with early surveillance biopsy, 81 were used as the discovery set (D) for identification of a gene set for prediction of early acute cellular rejection (EAR), i.e., before 6 months after transplant. We used 74 patients as the independent $\mathrm{V}$ set. Combined $\mathrm{V}$ and additional 80 patients with late surveillance biopsies (Late/L, $n=80$ ) cohorts (VL, $n=154$ ) were used to estimate association of gene set-derived risk score with late acute rejection (LAR) after 6 months, antibody-mediated rejection (ABMR), de novo donor-specific antibodies (DSAs) after transplant, or graft loss. All the patients had been followed up for at least 5 years or until graft loss or patient death.

to 23 optimal genes (Table 2) that accurately predicted EAR in the D set, with an AUC of 0.80 (95\% CI: 0.70 0.90 ) (Figure 3A, Supplemental Figure 6A). We further defined gene risk scores based on probability, stratifying patients into high, intermediate, and low risk (cutoff of 32 and -23) for EAR, with a positive predictive value (PPV) of 0.71 and a negative predictive value (NPV) of 0.88 for prediction of EAR (Supplemental Figure $6 \mathrm{~B}$ ). The risk score was similarly effective at predicting borderline or $1 \mathrm{~A}$ or greater AR episodes.

Next, the 23-gene set was tested on the independent V set $(n=74)$ using the gene risk score modeled on the D set and was found to have an AUC of 0.74 (95\% CI: 0.62-0.86) for prediction of EAR (black receiver operating characteristic [ROC] curve in Figure 3B and Supplemental Figure 7A). Combining the gene set with 3 demographic or clinical factors significantly associated with EAR (recipient age, kidney disease, and presence of anti-HLA antibody) improved the accuracy only slightly (AUC $=0.78$ with 95\% CI: 0.67-0.89) (Supplemental Figure 7B). Using the tertile cutoffs defined on the D set, the gene risk score performed similarly on the V set $(\mathrm{PPV}=0.70$ and $\mathrm{NPV}=0.88)$ (Figure $3 \mathrm{C})$.

Prediction of EAR using the gene set before transplant is independent of the donor characteristics. However, we found that when we analyzed the correlation between risk score and EAR in recipients with 4 or fewer HLA mismatches ( $n=45$ of the whole V cohort), the performance of the gene score was enhanced, predicting EAR with an AUC of 0.89 (95\% CI: 0.79-0.99), PPV of 0.75, and NPV of 1 (Figure 3, B and C).

Pretransplant blood transcriptomic signature is associated with $L A R, A B M R$, and de novo DSAs. We measured the association between the gene risk score and other clinical outcomes after transplant in 154 patients combining the V cohort $(n=74)$ and additional Genomics of Chronic Allograft Rejection (GoCAR) study patients with baseline whole-blood gene expression information and late surveillance biopsies between 6 and 24 months $(n=80)$. Patients in the $\mathrm{L}$ cohort had similar characteristics compared to those in the $\mathrm{D}$ and V cohorts (Table 1). We named the combined V + L cohort "VL cohort." In this cohort, the gene risk score was significantly associated with LAR $(P=0.041$, Supplemental Figure $7 \mathrm{C})$ and was even more significant when recipients with 5 or 6 HLA mismatches were excluded (Figure $4 \mathrm{~A} ; P=0.005 ; n=70$ [63\% VL]). 
Table 1. Demographic and clinical characteristics of patients

\begin{tabular}{|c|c|c|c|c|c|c|}
\hline Characteristics & Discovery set $(n=81)$ & Validation set $(n=74)$ & Late biopsy set $(n=80)$ & $P$ value $D$ versus $\mathbf{V}$ & $P$ value $\mathbf{V}$ versus $L$ & $P$ value $D$ versus $L$ \\
\hline Recipient age & $49 \pm 12.02$ & $49.7 \pm 14.11$ & $49.4 \pm 13.28$ & 0.7791 & 0.9053 & 0.8686 \\
\hline Recipient sex & & & & 0.5031 & 0.0983 & 0.0149 \\
\hline Male & & $50(61.73)$ & $50(67.57)$ & $64(80)$ & & \\
\hline Female & $31(38.27)$ & $24(32.43)$ & $16(20)$ & & & \\
\hline Recipient race & & & 0.0752 & 0.0528 & 0.9663 & \\
\hline White & $58(71.6)$ & $42(56.76)$ & $59(73.75)$ & & & \\
\hline Black or African American & $14(17.28)$ & $14(18.92)$ & $12(15)$ & & & \\
\hline Others & $9(11.11)$ & $18(24.32)$ & $9(11.25)$ & & & \\
\hline Dialysis (Y/N) & & & & 0.3037 & 0.0218 & 0.2160 \\
\hline $\mathrm{Y}$ & $63(77.78)$ & $63(85.14)$ & $55(68.75)$ & & & \\
\hline $\mathrm{N}$ & $18(22.22)$ & $11(14.86)$ & $25(31.25)$ & & & \\
\hline Anti-HLA antibody class I (Y/N) & & & & 0.1597 & 0.6973 & 0.0745 \\
\hline Y & $27(33.33)$ & $17(22.97)$ & $16(20)$ & & & \\
\hline $\mathrm{N}$ & $54(66.67)$ & $57(77.03)$ & $64(80)$ & & & \\
\hline Anti-HLA antibody class II (Y/N) & & & & 0.8415 & 0.8374 & 0.6900 \\
\hline Y & $17(20.99)$ & $14(18.92)$ & $14(17.5)$ & & & \\
\hline $\mathrm{N}$ & $64(79.01)$ & $60(81.08)$ & $66(82.5)$ & & & \\
\hline Induction type & & & & 0.1452 & $<0.0001$ & $<0.0001$ \\
\hline Non-lymphocyte depleting & $34(41.98)$ & $27(36.49)$ & $17(21.25)$ & & & \\
\hline Lymphocyte depleting & $27(33.33)$ & $18(24.32)$ & $56(70)$ & & & \\
\hline None & $20(24.69)$ & $29(39.19)$ & $7(8.75)$ & & & \\
\hline Kidney disease & & & & 0.5942 & 0.0545 & 0.4862 \\
\hline Diabetes mellitus & $24(29.63)$ & $25(33.78)$ & $22(27.5)$ & & & \\
\hline Glomerulonephritis & $21(25.93)$ & $18(24.32)$ & $13(16.25)$ & & & \\
\hline Hypertension & $16(19.75)$ & $9(12.16)$ & $20(25)$ & & & \\
\hline Polycystic kidney disease & $8(9.88)$ & $5(6.76)$ & $11(13.75)$ & & & \\
\hline Reflux & $3(3.7)$ & $6(8.11)$ & $1(1.25)$ & & & \\
\hline Others & 9 (11.11) & $11(14.86)$ & $13(16.25)$ & & & \\
\hline Donor age & $41.6 \pm 14.68$ & $42.9 \pm 15.92$ & $40.2 \pm 14.23$ & 0.5776 & 0.2758 & 0.5712 \\
\hline Donor sex & & & & 0.1464 & 0.0545 & 0.7528 \\
\hline Male & & $39(48.15)$ & $45(60.81)$ & $36(45)$ & & \\
\hline Female & $42(51.85)$ & $29(39.19)$ & $44(55)$ & & & \\
\hline Donor race & & & & 1 & 0.7579 & 0.8419 \\
\hline White & $66(81.48)$ & $61(82.43)$ & $63(78.75)$ & & & \\
\hline Black or African American & $6(7.41)$ & $5(6.76)$ & $8(10)$ & & & \\
\hline Others & $9(11.11)$ & $8(10.81)$ & $9(11.25)$ & & & \\
\hline Deceased donor $(\mathrm{Y} / \mathrm{N})$ & & & & 0.5196 & 0.0012 & 0.007 \\
\hline Y & $45(55.56)$ & $45(60.81)$ & $27(33.75)$ & & & \\
\hline $\mathrm{N}$ & $36(44.44)$ & $29(39.19)$ & $53(66.25)$ & & & \\
\hline HLA overall mismatch & & & & 0.5753 & 0.3563 & 0.9044 \\
\hline mismatch (0) & $10(12.35)$ & $9(12.16)$ & $7(8.75)$ & & & \\
\hline mismatch (1-2) & $12(14.81)$ & $6(8.11)$ & $13(16.25)$ & & & \\
\hline mismatch (3-4) & $33(40.74)$ & $30(40.54)$ & $35(43.75)$ & & & \\
\hline mismatch (5-6) & $26(32.1)$ & $29(39.19)$ & $25(31.25)$ & & & \\
\hline HLA-A mismatch & & & & 0.9775 & 0.5974 & 0.445 \\
\hline mismatch (0) & $19(23.4568)$ & $16(21.6216)$ & $13(16.6667)$ & & & \\
\hline mismatch (1) & $38(46.9136)$ & $36(48.6486)$ & $44(56.4103)$ & & & \\
\hline mismatch (2) & 24 (29.6296) & $22(29.7297)$ & $21(26.9231)$ & & & \\
\hline HLA-B mismatch & & & & 0.5607 & 0.7886 & 0.8231 \\
\hline mismatch (0) & $14(17.7215)$ & $11(14.8649)$ & $11(14.2857)$ & & & \\
\hline mismatch (1) & $27(34.1772)$ & $21(28.3784)$ & $26(33.7662)$ & & & \\
\hline mismatch (2) & $38(48.1013)$ & $42(56.7568)$ & $40(51.9481)$ & & & \\
\hline HLA-DR mismatch & & & & 0.8053 & 0.8687 & 0.4331 \\
\hline mismatch (0) & $22(27.1605)$ & $17(22.973)$ & $14(18.9189)$ & & & \\
\hline mismatch (1) & $31(38.2716)$ & $28(37.8378)$ & $29(39.1892)$ & & & \\
\hline mismatch (2) & $28(34.5679)$ & $29(39.1892)$ & $31(41.8919)$ & & & \\
\hline Race mismatch $(\mathrm{Y} / \mathrm{N})$ & & & & 0.1029 & 0.0041 & 0.2988 \\
\hline Y & $17(20.99)$ & 25 (33.78) & $11(13.75)$ & & & \\
\hline $\mathrm{N}$ & $64(79.01)$ & $49(66.22)$ & $69(86.25)$ & & & \\
\hline CIT min (within deceased donor patients) & $777.6 \pm 400.76$ & $849.8 \pm 440.24$ & $819 \pm 372.73$ & 0.4183 & 0.7523 & 0.6594 \\
\hline $\operatorname{DGF}(\mathrm{Y} / \mathrm{N})$ & & & & 1 & 0.2399 & 0.1759 \\
\hline Y & $15(18.52)$ & $13(17.57)$ & $8(10)$ & & & \\
\hline $\mathrm{N}$ & $66(81.48)$ & $61(82.43)$ & $72(90)$ & & & \\
\hline DSAs at baseline $(\mathrm{Y} / \mathrm{N})$ & & & & 0.3881 & 0.2294 & 0.7441 \\
\hline $\mathrm{Y}$ & $5(6.49)$ & $8(11.27)$ & $4(5.06)$ & & & \\
\hline $\mathrm{N}$ & $72(93.51)$ & $63(88.73)$ & 75 (94.94) & & & \\
\hline
\end{tabular}

All patients were BK negative. Numbers are presented as mean \pm SD or count (percentage \%). CIT, cold ischemia time; DCF, delayed graft function. 
A

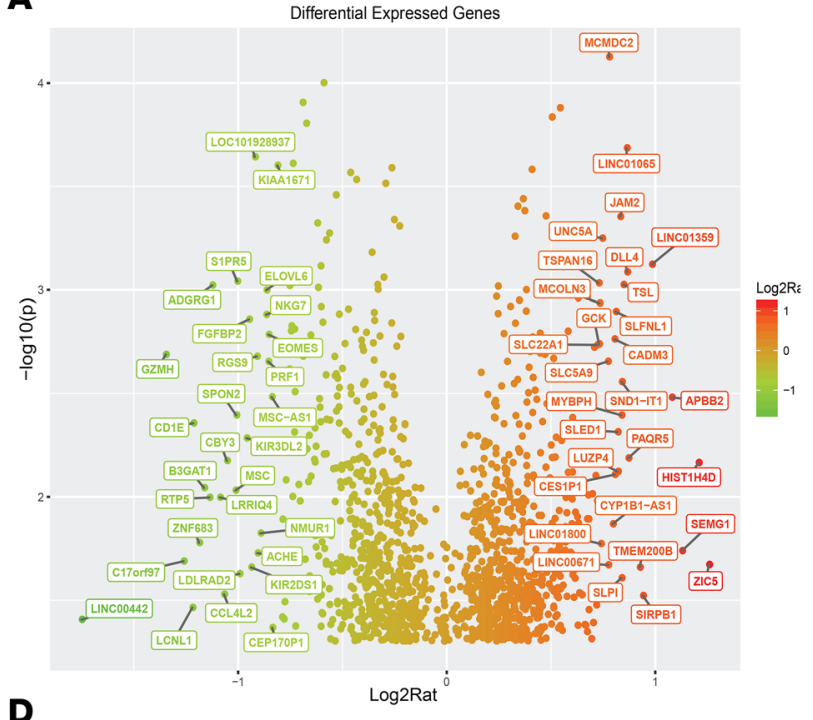

D

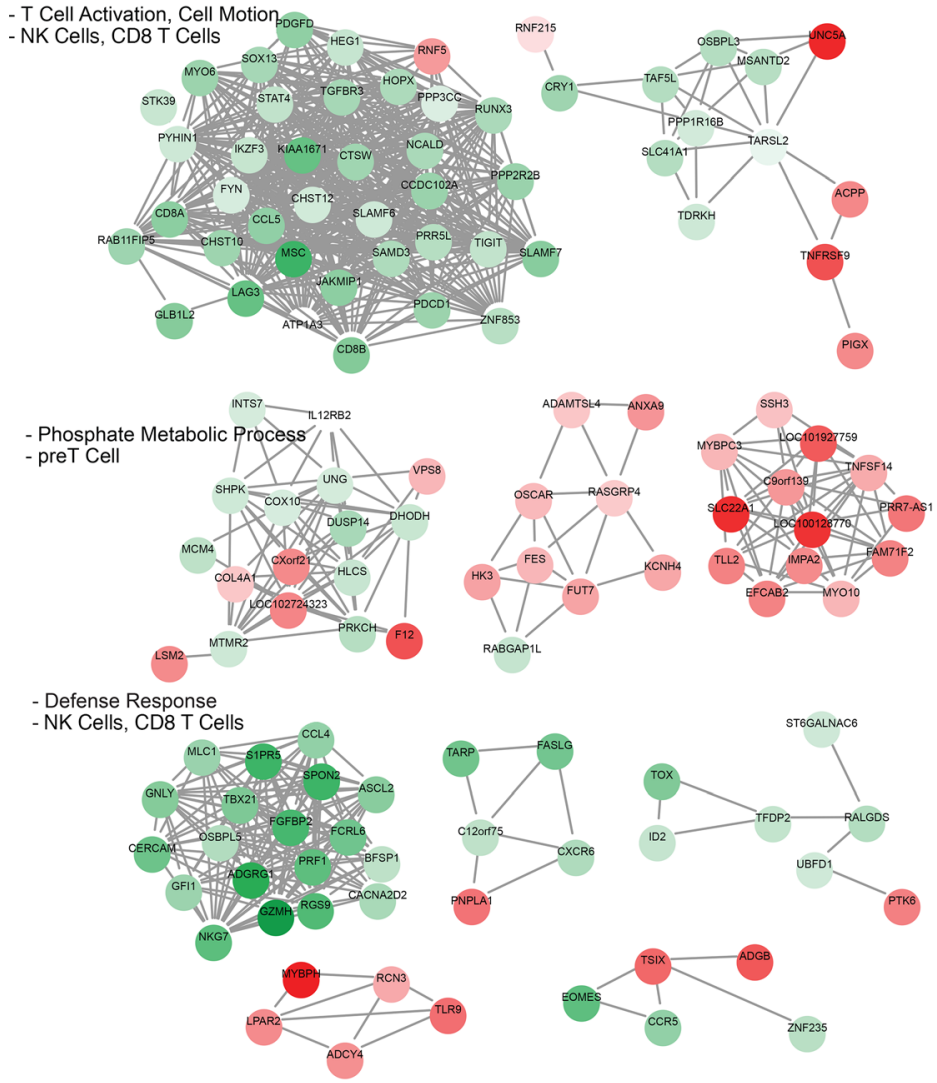

B
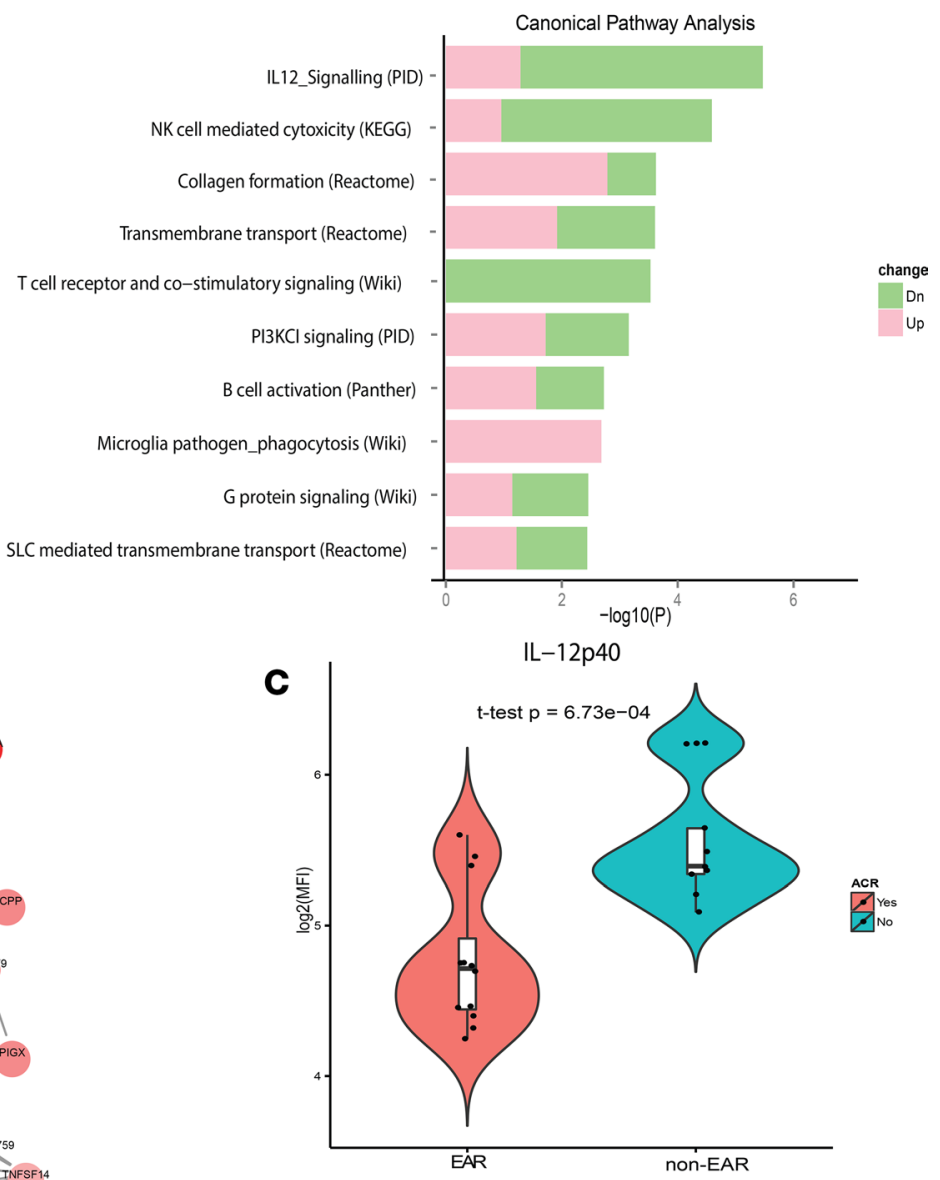

E
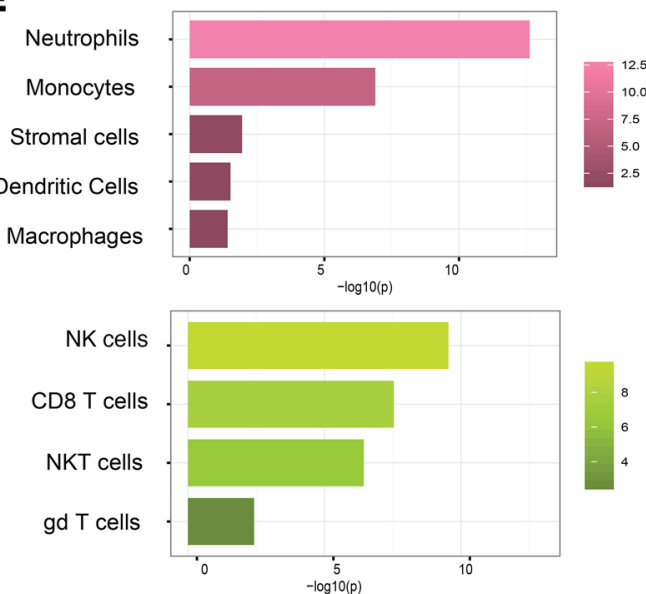

Figure 2. Pretransplant whole-blood transcriptomic signatures of the patients with or without EAR. (A) The volcano plot of differentially expressed genes (DEGs) between the recipients who developed or did not develop EAR. The $x$ axis depicts the $\log _{2}$ ratio of gene expression, and the $y$ axis depicts the $-\log _{10}$ of limma $P$ test (ref. 50). The top upregulated or downregulated genes are labeled with boxes. (B) The bar charts of enriched canonical pathways by enrichment analysis on DEGs from multiple pathway databases (indicated in parentheses). The bar represents -log10 $P$ value of enrichment significance of gene pathways by Fisher's exact test; the lengths of red and green bars represent the percentage of upregulated and downregulated genes, respectively. (C) The violin plot of the distribution of pretransplant serum IL-12p40 expression between those recipients who developed EAR (red bar) and those who did not (blue bar). (D) Core co-regulation modules identified by Markov Cluster Algorithm from the correlation matrix of DEGs in the recipients with EAR after transplant. The red and green nodes indicate the upregulated and downregulated genes, respectively. (E) The bar charts of enriched immune cell types for upregulated (purple bars) and downregulated (green bars) genes based on immune cell-specific genes identified from ImmGen database. The $x$ axis depicts $-\log _{10} P$ value of enrichment significance of immune cell types. gd, gamma delta. 
Table 2. The list of the 23-gene set

\begin{tabular}{|c|c|c|c|}
\hline Symbol & Name & $P$ (discovery) & $\log _{2}$ ratio (discovery) \\
\hline GZMH & Granzyme H & 0.0021 & -1.35 \\
\hline ADGRG1 & Adhesion G protein-coupled receptor G1 & 0.0010 & -1.12 \\
\hline FGFBP2 & Fibroblast growth factor binding protein 2 & 0.0014 & -0.95 \\
\hline NKG7 & NK cell granule protein 7 & 0.0013 & -0.87 \\
\hline PRF1 & Perforin 1 & 0.0022 & -0.86 \\
\hline TARP & T cell receptor $-\gamma$ alternate reading frame protein & 0.0024 & -0.76 \\
\hline FCRL6 & Fc receptor-like 6 & 0.0016 & -0.75 \\
\hline FASLG & Fas ligand & 0.0002 & -0.74 \\
\hline TBX21 & T-box 21 & 0.0021 & -0.69 \\
\hline TOX & Thymocyte selection associated high mobility group box & 0.0002 & -0.68 \\
\hline LDOC1L & LDOC1 like & 0.0005 & -0.57 \\
\hline CCDC102A & Coiled-coil domain containing $102 \mathrm{~A}$ & 0.0026 & -0.54 \\
\hline HOPX & HOP homeobox & 0.0016 & -0.44 \\
\hline PRKCH & Protein kinase $[-\eta$ & 0.0028 & -0.39 \\
\hline SLC25A34 & Solute carrier family 25 member 34 & 0.0001 & 0.5 \\
\hline F12 & Coagulation factor XII & 0.0016 & 0.58 \\
\hline
\end{tabular}

We next tested the association between the gene risk score and ABMR and/or de novo DSAs, 2 major risk factors for graft loss $(19,20)(\mathrm{VL}, n=154)$. Twenty-three patients who developed ABMR $(n=$ $7)$, de novo DSAs $(n=12)$, or both $(n=4)$ had a significantly higher risk score than the recipients without clinical events after transplant $\left(P=5.17 \times 10^{-4}\right.$, Supplemental Figure 7D). Among these 23 patients, 21 patients had a high or intermediate risk score, and only 2 patients had a low risk score (Supplemental Figure 7E). If only the patients with 4 or fewer HLA mismatches were considered, the significance of risk scores of 12 patients with ABMR or de novo DSAs was even higher $\left(P=3.07 \times 10^{-8}\right.$, Figure $\left.4 \mathrm{~B}\right)$, all with high or intermediate risk score (Figure $4 \mathrm{C}$ ). These data demonstrated that the gene risk score can identify patients at risk for significant immunological events after transplant.

Pretransplant blood transcriptomic signature is associated with death-censored graft loss. When patients were stratified into tertiles based on individual risk scores, the high-risk group had a significantly higher rate of death-censored graft loss in the VL cohort ( $n=154 ; P=0.043$; Supplemental Figure 7F). When we repeated the analyses in recipients of grafts with no more than 4 HLA mismatches, the gene risk score was able to better stratify the risk of graft loss (Figure $4 \mathrm{D} ; n=100\left[65 \%\right.$ of VL]; $P=3.09 \times 10^{-4}$ ).

Overall, the risk score accurately predicted graft loss at any time (AUC 0.804-0.904) within patients with no more than 4 HLA mismatches. When only grafts lost within 2 years after transplant were considered, the AUC for patients with no more than 4 HLA mismatches was 0.904 (95\% CI: 0.84-0.97), while the AUC for graft loss before 5 years was 0.820 (95\% CI: 0.69-0.95) (Figure 4E).

In summary, none of the patients with no more than 4 HLA mismatches and low risk score in the VL cohort had any major immunological event or graft loss at 2 years of follow-up (Figure $4 \mathrm{~F} ; n=$ $100 ; \mathrm{PPV}=0.75$, and NPV $=1.00$ ).

Recipient age and kidney disease are associated with pretransplant gene risk score but do not predict EAR. We next tested the association between recipient pretransplant characteristics and gene risk score and found that recipient age and etiology of kidney diseases were significantly associated with the risk score in the VL cohort ( $n=154$; $P=0.011$, and $P=0.024$, respectively; Supplemental Table 4), with younger recipients or those with a diagnosis of glomerulonephritis having a higher risk score and higher rate of $\operatorname{EAR}(P=0.001$, and $P=0.007$, respectively; Supplemental Figure 8, A and B). However, recipient age and kidney diseases in combination failed to predict EAR, nor did they increase the predictive power significantly when combined with the gene risk score (AUC increased from 0.74 for gene risk score alone to 0.78 when combined) (Supplemental Figure 7B). 
A

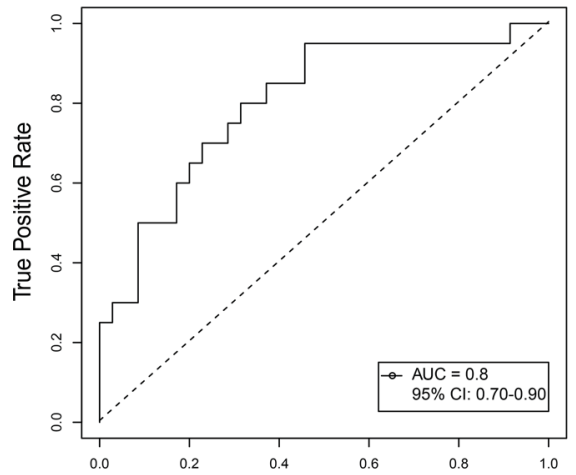

B

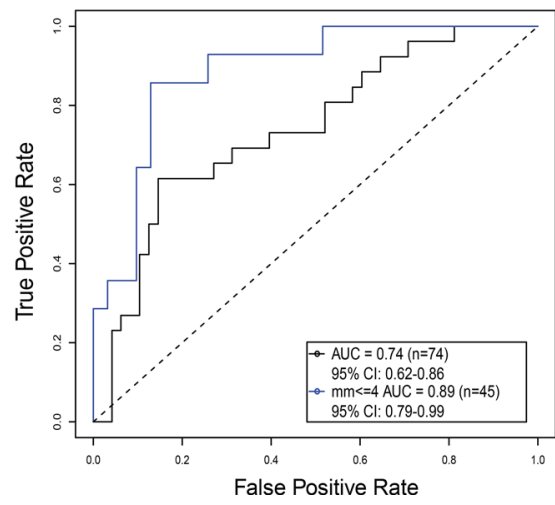

C
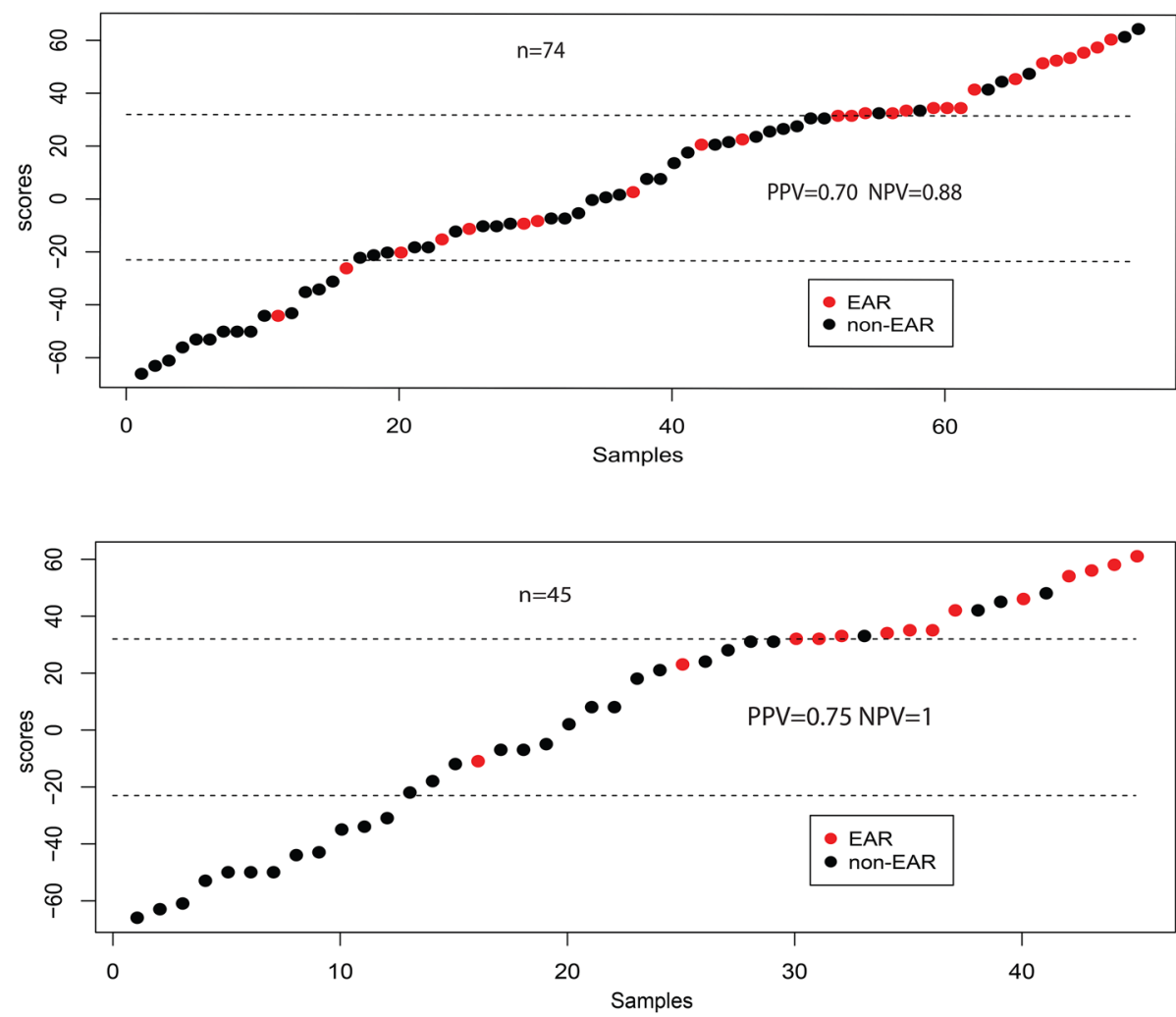

Figure 3. Identification of 23-gene set for prediction of EAR and graft loss. (A) The ROC curve for prediction of EAR with risk score in the $D$ set $(n=81$, and AUC $=0.80$ ). (B) The ROC curve of prediction of EAR in the $V$ set. The black line represents all patients $(n=74$, and $A U C=0.74)$; the blue line indicates patients with no more than 4 HLA mismatches $(n=45$, and AUC $=0.89)$. (C) The dot plots of the risk scores for the patients in $V$ set are in the top plot $(n=$ $74, \mathrm{PPV}=0.70$, and NPV $=0.88$ at tertile cutoffs), and in the lower plot are V patients with no more than $4 \mathrm{HLA}$ mismatches $(n=45, \mathrm{PPV}=0.75$, and NPV $=$ 1 at tertile cutoffs). Red dots represent patients with EAR.

\section{Discussion}

We found a 23-gene transcriptional signature in pretransplant whole blood that accurately predicts EAR in kidney transplant recipients. Because of the strong association between EAR and both LAR and graft loss, the transcriptional signature was also a strong predictor of both of these outcomes. Our results are the first to our knowledge to describe a pretransplant gene risk profile that has the potential to be applied broadly across renal transplant recipients. This moves beyond the current approach in which high-risk recipients are identified using clinical information predominately related to DSAs and previous exposure to alloantigen, which fails to differentiate risk in the wider group of recipients. Although the pretransplant blood transcriptional signature was associated with recipient age and kidney disease, these clinical characteristics taken alone or in combination did not predict EAR and did not significantly increase the predictive power of the gene risk score.

The concept that a gene signature in the blood can stratify the immunological risk of individuals receiving a graft is consistent with data by others showing patterns of gene expression by peripheral blood mononuclear cells to risk stratify and diagnose patients with autoimmune diseases $(21,22)$ or cancer $(23)$. Our transcriptomic analysis of pretransplant blood revealed the downregulation of $\mathrm{NK}$ and $\mathrm{CD} 8^{+} \mathrm{T}$ cell signatures in subjects at higher risk for EAR. However, the total $\mathrm{CD} 8^{+} \mathrm{T}$ cell numbers alone pretransplant did not predict $\operatorname{EAR}(24,25)$, indicating that the gene risk score conveys additional information that cannot be inferred by simple measurement of circulating cells. Our findings are in keeping with previous published data indicating that NK cells prevent autoimmune disease by inhibiting activation of antigen-presenting cells (26). Reduced numbers of circulating NK cells have been detected in patients with systemic lupus erythematosus and other autoimmune diseases compared with healthy controls (26-28). Reduced pretransplant serum levels of IL-12, a major driver for NK cell activation (29), may account for the lower numbers of circulating NK cells in high-risk patients, supporting previous observations that, in contrast with its 
A

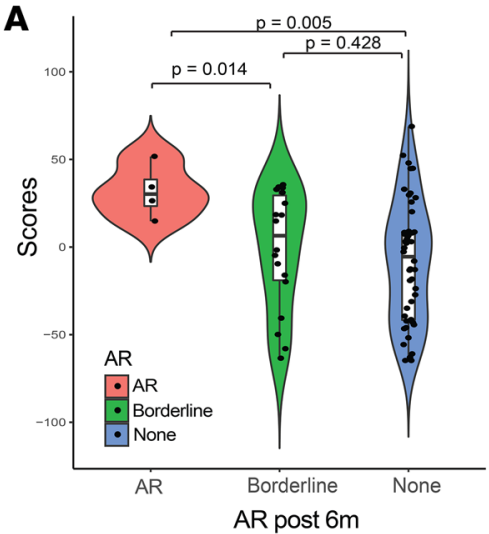

B

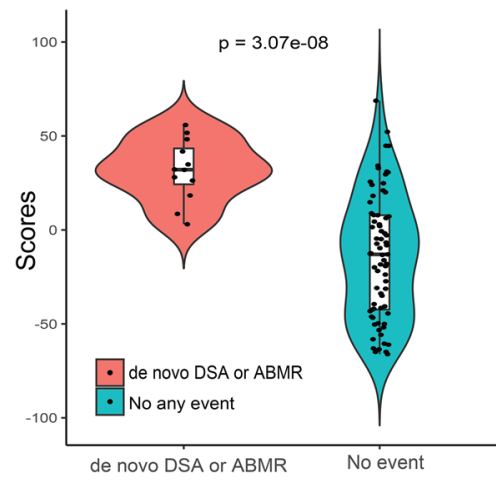

C

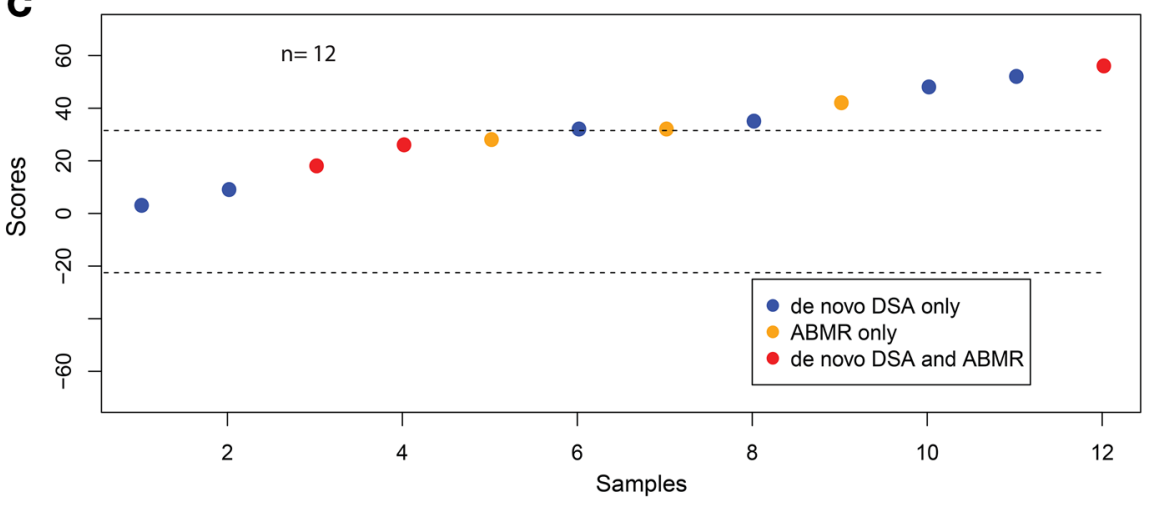

$\mathbf{E}$
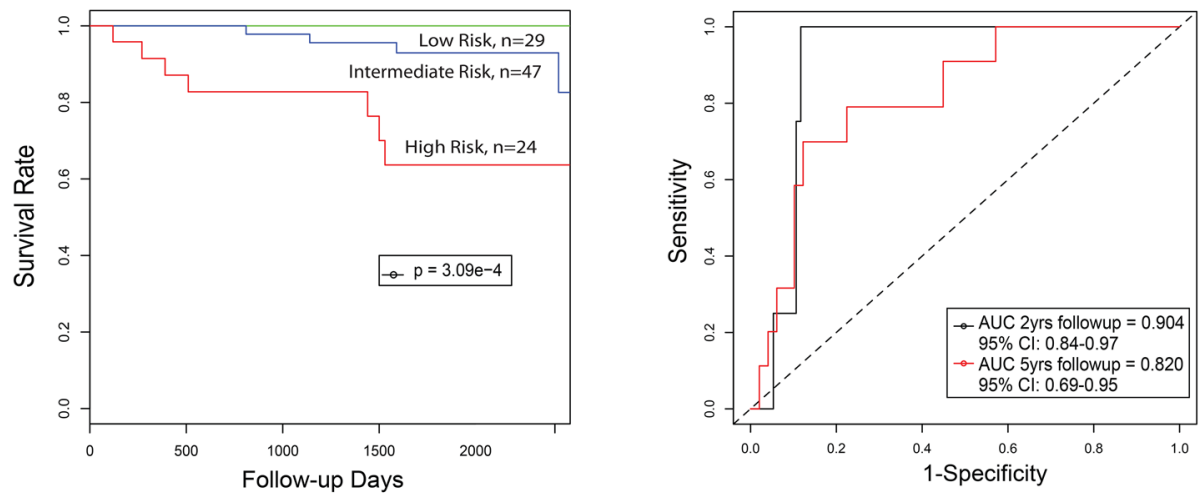

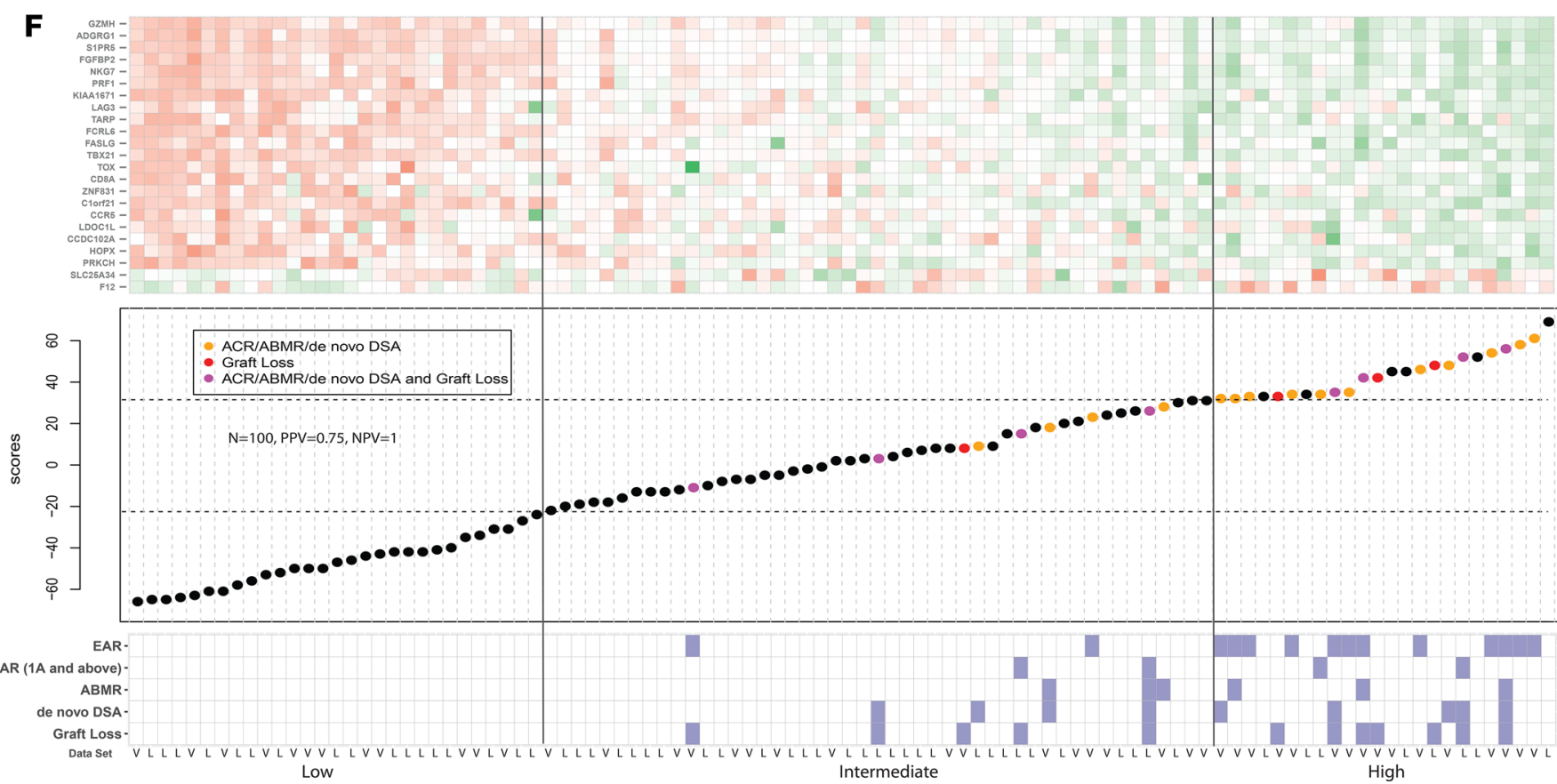

Figure 4. Association of gene risk score with clinical outcomes after transplant in VL cohort with no more than 4 HLA mismatches ( $n=100)$. (A) The violin plot of distribution of risk scores among the patients with AR at borderline and $1 A$ and above, and with no AR beyond 6 months after transplant; $P$ values are significant between the AR and no AR groups ( $t$ test $P=0.005$ ) and between the AR and borderline groups $(t$ test $P=0.014)$. (B) The violin plot of distribution of risk scores between the patients who developed ABMR or de novo DSAs and those without clinical events $\left(t\right.$ test $\left.P=3.07 \times 10^{-8}\right)$. (C) The dot plot of the gene risk scores of 12 patients who developed ABMR (gold), de novo DSAs (blue), or both (red). (D) The Kaplan-Meier curve of graft loss with the kidney transplant recipients stratified by high, intermediate, or low risk based on tertile cutoffs (log-rank test $P=3.09 \times 10^{-4}$ ). (E) The ROC curve for prediction of graft loss at 2 (black curve, AUC $=0.904$ ) or 5 (red curve, $A U C=0.820$ ) years after transplant. (F) Summary plots of association of gene risk score with clinical outcomes of kidney transplant patients in $\mathrm{V}$ and Late/L sets: the heatmap on the top shows the expression of the 23-gene set of the patients with low to high risk scores. The middle dot plot displays the risk scores of the patients who developed graft loss (red), immunological events (AR/ABMR/de novo DSAs, gold), or both (purple). The bottom indicates the clinical events for each patient. 
proinflammatory effects, IL-12 is associated with prolonged graft survival (30). CD8 ${ }^{+} \mathrm{NK}$ T cells with regulatory properties have also been described (31), and their reduced number may expose patients to higher risk of rejection and a subsequent graft failure. Finally, we found that patients with high gene risk score had a significant reduction in the expression of programmed cell death 1 gene (not shown), a major regulator of $\mathrm{T}$ cell exhaustion (32) and of regulatory function (33), both important functions in preventing AR.

Currently, antirejection therapy for most kidney transplant recipients is relatively standard within each center with most patients receiving similar maintenance immunosuppression. Patients perceived to be at higher risk for AR generally receive more intense induction therapy, but the criteria to identify those patients are very broad and fail to differentiate the risk among standard patients. This is a major issue because an increased cumulative dose of immunosuppression is associated with higher risk of infections and neoplasms (34). The ability to target those who truly need heightened immunosuppression has the potential to decrease morbidity and mortality after transplantation overall.

In the present study, demographic and pretransplant clinical characteristics were associated with but unable to accurately predict EAR, making them of little utility in determining the need for increased immunosuppression. In contrast, the transcriptional profile of whole-blood cells at the time of transplant was able to identify patients at increased risk for EAR. Importantly, our transcriptional profile, developed to detect cellular EAR, was also associated with increased risk for de novo DSAs and/or ABMR. This finding is in line with recent evidence that $\mathrm{AMBR}$ and $\mathrm{T}$ cell receptor share many molecular transcripts $(35,36)$. Our data suggest that a low risk score at the time of transplant identifies individuals whose chances of developing immunological events damaging to the graft are remote, thereby suggesting that they may need lower doses of potentially toxic antirejection therapies aimed at preventing acute rejection.

Our transcriptional profile was able to quantify the immunological risk independent of donor information, suggesting the existence of an intrinsic "inflammatory phenotype" that could be captured by the RNA sequencing analyses. Our study showed that patients with immune-mediated glomerulonephritides had a higher gene risk score compared patients with no immune-mediated conditions, which is in line with data from other studies in autoimmune disorders, where disease activity was associated with changes in peripheral gene expression profiles (37). The ability to capture such an "inflammatory phenotype" is important because it has the potential to identify patients at highest need for increased immunosuppression to prevent rejection. The risk score is not independent of donor factors, but rather both must be considered. Patients with a low risk score who received a graft with more than 4 mismatches behaved as an intermediate- or high-risk patient. This suggests that the transcriptional signature where appropriate may also be used to guide decisions regarding organ allocation; for example, in patients with low transcriptional risk, finding a living or deceased donor with no more than 4 HLA mismatches may minimize the risk of EAR. Recent data indicate that more sophisticated strategies to assess the conformational mismatch between donor and recipient HLA alleles may further refine the risk of developing de novo DSAs, ABMR, or early graft loss (38-40). These approaches could be used in parallel with our gene risk score to further refine its predictive accuracy.

Our study has some limitations. Patient immunosuppression was not standardized. Although this may have biased the results, this "real-life" approach is important because it shows that the gene score is applicable in clinical settings across immunosuppression protocols. DSA monitoring was not done as part of the study at the time of surveillance biopsies but according to clinical practice. Although we acknowledge that lack of a common approach in DSA measurements may have biased the results, we also contend that such an approach increases the clinical significance of our positive finding.

The majority of EAR in our cohort was detected at surveillance biopsy and was scored as borderline. Consistent with data by others $(41,42)$, our current results demonstrate that borderline EARs increase the risk of late rejections and graft loss. Therefore, our gene signature is relevant and may allow the identification of patients who could benefit the most from induction therapy or increased maintenance immunosuppression. This assay may also allow identification of patients at low risk for EAR, where surveillance biopsies may be avoided (43-45).

In summary, we have identified a 23-gene pretransplant signature that can accurately predict EAR and that is associated with LAR and graft loss. Our findings warrant further evaluation of the gene risk score in additional transplant cohorts, with the ultimate goal of determining its role in guiding immunosuppression administration on an individual basis with the aim of minimizing complications and maximizing graft survival. 


\section{Methods}

Patients. The GoCAR study is a prospective, multicenter study aimed at investigating the genetics and genomics associated with the development of allograft rejection or injury in kidney transplant recipients. A subset of patients in the GoCAR cohort, those at Mount Sinai and Westmead, had protocol biopsies at multiple time points $(0,1,3,6,12$, and 24 months) for examining gene expression. The remainder of sites had biopsies performed at 0 and 24 months to allow for a larger population of patients for genetic and genomic analyses. In addition, later in the study, 2 sites started performing surveillance biopsies as routine care on all patients. When these patients were enrolled in the GoCAR, a core was taken for the study in addition to the 24-month core per GoCAR protocol. The details of patient enrollment criteria and study design for the GoCAR study have been previously described (7). The patients with DSAs requiring desensitization were not included in the GoCAR. Immunosuppressive therapy included lymphocyte-depleting or non-lymphocyte-depleting induction or no induction (Table 1), and maintenance immunosuppression consisted of calcineurin inhibitors and mycophenolate mofetil, with or without steroids (7). BK nephropathy (BKN) can present with viral cytopathic changes in tubular cells associated with tubulointerstitial nephritis that is indistinguishable from cellular rejection (46) while immune activation in peripheral blood may actually reflect decreased activity in contrast with increased activity in acute rejection (47). Because of these potentially confounding factors, the patients with biopsy-proved BKN were excluded from this study.

A total of 289 patients enrolled, had serial surveillance biopsy, and had baseline whole-blood RNA samples available. Ten of these patients had BKN and were excluded from this analysis because of the potential overlap between BKN and ACR. RNA of sufficient quality (RNA integrity number $\geq$ 6) was available from 235 patients for RNA sequencing. Of these 235 patients, 155 had pretransplant biopsies and serial after transplant surveillance biopsies up to 24 months after transplant per protocol. An additional 80 patients had only later surveillance biopsies ( $>6$ months). The reasons for the lack of the earlier time point biopsies were clinical site protocol, medical reasons, declining by patient, loss of allograft, and being lost to follow-up. For determination of EAR (the primary endpoint) in this study, 155 patients were randomly assigned into $\mathrm{D}(n=81)$ and $\mathrm{V}(n=74)$ sets. To study the association of the gene risk score with other endpoints, such as LAR (>6 months), graft loss, ABMR, and de novo DSAs, we combined V set $(n=74)$ with the additional 80 patients (Late/L, $n=80$ ) for whom baseline blood and late surveillance biopsies between months 6 to 24 after transplant were taken. This group of 154 patients $(74+80)$ was referred to as "VL set." The D set was not included to avoid overfitting (Figure 1). All the patients had been followed up for at least 5 years or until graft loss or patient death. Pretransplant donor and recipient characteristics were compared among the 3 populations within the RNA sequencing cohort (the D, V, and Late/L sets) (Table 1).

The diagnosis of AR was made using Banff 2013 Classification $(48,49)$ on all clinically indicated and surveillance biopsies and included borderline subclinical rejection. The diagnosis of AR, including the borderline and 1A and above, was reported by the central Massachusetts General Hospital pathological core. EAR and LAR were defined as cellular rejection within or after 6 months following transplant, respectively, with or without concurrent features of ABMR or de novo DSAs. DSAs were measured by Luminex before transplant and when clinically indicated thereafter, according to center practice.

$R N A$ sequencing experiments and data analysis. The details of RNA sequencing experiments and data analysis are provided in Supplemental materials, and the analysis workflow is depicted in Supplemental Figure 2. Briefly, total RNA was extracted from whole blood drawn from the recipients before transplant, and mRNA sequencing was performed on an Illumina HiSeq 4000 sequencer. After a series of read quality control, mapping, and normalization steps on raw sequence reads, the expression data between EAR and non-EAR groups in the D set were compared to initially identify DEGs, and enrichment analysis of canonical pathway/gene ontology/immune cell types of DEGs was performed. Luminex protein assays using an immune profile panel were performed on the serum of patients to investigate the correlation of cytokine expression with dysregulated pathways. The correlation network analysis was also performed on the DEGs to build the expression correlation matrix to identify core co-regulation modules. The immune cell populations of the blood were deconvoluted from the bulk transcriptomic profiles using a published expression matrix of sorted human immune cells (18).

To identify an optimal gene set to predict EAR, a focus gene set specifically associated with EAR was identified under the criteria described in Supplemental materials. Using the cumulative risk score that summarizes the logic numbers for comparison of gene expression to the median value of the EAR or nonEAR group ( 1 if the expression favors EAR group, -1 if the expression favors non-EAR group, or 0 if the 
expression is between the EAR and non-EAR groups), which was weighted by the significance $P$ value of the difference between the EAR and non-EAR groups (refer to the formula for risk score calculation in Supplemental materials), a minimal gene set with the best predictive area under ROC curve was identified in the $\mathrm{D}$ set from the initial focus gene set with iteration of forward selection steps. The prediction of EAR by the final gene set was validated in the $\mathrm{V}$ data set, and the association of the gene set with LAR, ABMR, de novo DSAs, and/or graft loss was determined using the VL set. The prediction accuracy was further evaluated in the recipient subgroup with no more than 4 HLA mismatches.

Last, the demographic and pretransplant clinical characteristics of recipients who were associated with the gene set-derived risk score were examined in the VL set.

The RNA sequencing data are posted in the National Center for Biotechnology Information's Gene Expression Omnibus database (GEO GSE112927).

Clinical statistical analyses. In total, 155 patients (81 in D set and 74 in V set) underwent surveillance biopsy before 6 months. We tested for correlation between EAR and LAR by Fisher's exact test and graft loss by log-rank and Gehan-Breslow-Wilcoxon test using $\mathrm{R}$ package. The pretransplant demographic or clinical characteristics that were significantly associated with EAR were determined and further investigated if they could predict EAR.

The pretransplant demographic or clinical characteristics among RNA sequencing cohorts were compared by $t$ test for continuous variables or Fisher's test for categorical variables using R statistical packages.

Study approval. The study was carried out in accordance with the principles of the Declaration of Helsinki, and all procedures were approved by the Human Subjects Committee at participating sites. All participants provided informed consent.

\section{Author contributions}

WZ led the project, designed the study, analyzed and interpreted genomic data, and drafted the manuscript. ZY performed genomic data analysis (major contribution) and interpretation and drafting of Supplemental materials. C. Wei performed sample preparation and quality control. KLK analyzed and interpreted clinical data. ZS analyzed genomic data. CX performed clinical data management and querying. C. Woytovich performed sample preparation. SF analyzed and interpreted clinical data. LG performed patient enrollment and follow-up. MCM interpreted clinical data and critically reviewed the manuscript. CM and NN performed pathology reporting. MDS performed patient enrollment and follow-up. AD performed patient enrollment and follow-up. SIA provided study design discussion and critical review of the manuscript. IAR performed pathology reporting. RS performed pathology reporting. PJO provided interpretation of clinical data, discussion of study design, and critical revision of the manuscript. RC performed pathology reporting. PC performed analysis and interpretation of clinical data, along with drafting of the manuscript. BM, the principal investigator, performed study conception and design, along with drafting of the manuscript.

\section{Acknowledgments}

We thank Joel Dudley and Li Li for suggestions for genomic data processing and analysis. We thank the Genomics and Epigenomics Core Facility at Weill Cornell Medical Center for RNA sequencing experiments and the Scientific Computing division at the Icahn School of Medicine at Mount Sinai for providing computational resource. This work is a substudy of the GoCAR study sponsored by NIH 5U01AI070107-03.

Address correspondence to: Barbara Murphy, Division of Nephrology, Department of Medicine, Icahn School of Medicine at Mount Sinai, One Gustave L. Levy Place, Box 1243, New York, New York 10029, USA. Phone: 212.241.8001; Email: barbara.murphy@mssm.edu.

\footnotetext{
1. Abecassis M, et al. Kidney transplantation as primary therapy for end-stage renal disease: a National Kidney Foundation/Kidney Disease Outcomes Quality Initiative (NKF/KDOQITM) conference. Clin J Am Soc Nephrol. 2008;3(2):471-480.

2. E1 Ters M, et al. Kidney allograft survival after acute rejection, the value of follow-up biopsies. Am J Transplant. 2013;13(9):2334-2341

3. [No authors listed]. United Network for Organ Sharing database. https://unos.org/data/. Accessed May $20,2019$.

4. Morales JM, et al. Risk factors for graft loss and mortality after renal transplantation according to recipient age: a prospective multicentre study. Nephrol Dial Transplant. 2012;27(suppl 4):iv39-iv46.

5. Jevnikar AM, Mannon RB. Late kidney allograft loss: what we know about it, and what we can do about it. Clin J Am Soc Nephrol. 2008;3 Supp1 2:S56-S67.
} 
6. Riella LV, Djamali A, Pascual J. Chronic allograft injury: Mechanisms and potential treatment targets. Transplant Rev (Orlando). 2017;31(1):1-9.

7. O'Connell PJ, et al. Biopsy transcriptome expression profiling to identify kidney transplants at risk of chronic injury: a multicentre, prospective study. Lancet. 2016;388(10048):983-993.

8. Gaston RS, et al. Late graft failure after kidney transplantation as the consequence of late versus early events. Am J Transplant. 2018;18(5):1158-1167.

9. Krishnan AR, et al. Prolonged ischemic time, delayed graft function, and graft and patient outcomes in live donor kidney transplant recipients. Am J Transplant. 2016;16(9):2714-2723.

10. Nankivell BJ, Borrows RJ, Fung CL, O'Connell PJ, Allen RD, Chapman JR. The natural history of chronic allograft nephropathy. N Engl J Med. 2003;349(24):2326-2333.

11. Gago M, Cornell LD, Kremers WK, Stegall MD, Cosio FG. Kidney allograft inflammation and fibrosis, causes and consequences. Am J Transplant. 2012;12(5):1199-1207.

12. Angeletti A, Cravedi P. Looking into the graft without a biopsy: biomarkers of acute rejection in renal transplantation. Contrib Nephrol. 2017;190:181-193.

13. Menon MC, Murphy B, Heeger PS. Moving biomarkers toward clinical implementation in kidney transplantation. $J A m$ Soc Nephrol. 2017;28(3):735-747.

14. Yabu JM, Siebert JC, Maecker HT. Immune profiles to predict response to desensitization therapy in highly HLA-sensitized kidney transplant candidates. PLoS One. 2016;11(4):e0153355.

15. Poggio ED, Clemente M, Hricik DE, Heeger PS. Panel of reactive T cells as a measurement of primed cellular alloimmunity in kidney transplant candidates. J Am Soc Nephrol. 2006;17(2):564-572.

16. Subramanian A, et al. Gene set enrichment analysis: a knowledge-based approach for interpreting genome-wide expression profiles. Proc Natl Acad Sci U S A. 2005;102(43):15545-15550.

17. Heng TS, Painter MW, Immunological Genome Project Consortium. The Immunological Genome Project: networks of gene expression in immune cells. Nat Immunol. 2008;9(10):1091-1094.

18. Newman AM, et al. Robust enumeration of cell subsets from tissue expression profiles. Nat Methods. 2015;12(5):453-457.

19. Loupy A, et al. Complement-binding anti-HLA antibodies and kidney-allograft survival. N Engl J Med. 2013;369(13):1215-1226

20. Otten HG, Verhaar MC, Borst HP, Hené RJ, van Zuilen AD. Pretransplant donor-specific HLA class-I and -II antibodies are associated with an increased risk for kidney graft failure. Am J Transplant. 2012;12(6):1618-1623.

21. Maas K, et al. Cutting edge: molecular portrait of human autoimmune disease. J Immunol. 2002;169(1):5-9

22. Mesko B, Poliska S, Nagy L. Gene expression profiles in peripheral blood for the diagnosis of autoimmune diseases. Trends Mol Med. 2011;17(4):223-233.

23. Reis-Filho JS, Pusztai L. Gene expression profiling in breast cancer: classification, prognostication, and prediction. Lancet. 2011;378(9805):1812-1823.

24. Vondran FW, et al. Pre-transplant immune state defined by serum markers and alloreactivity predicts acute rejection after living donor kidney transplantation. Clin Transplant. 2014;28(9):968-979.

25. Dedeoglu B, et al. Loss of CD28 on peripheral T cells decreases the risk for early acute rejection after kidney transplantation. PLoS One. 2016;11(3):e0150826.

26. Schleinitz N, Vély F, Harlé JR, Vivier E. Natural killer cells in human autoimmune diseases. Immunology. 2010;131(4):451-458.

27. Erkeller-Yuksel FM, Lydyard PM, Isenberg DA. Lack of NK cells in lupus patients with renal involvement. Lupus. 1997;6(9):708-712.

28. Wolfe F, Hawley DJ, Wilson K. The prevalence and meaning of fatigue in rheumatic disease. J Rheumatol. 1996;23(8):1407-1417

29. Ferlazzo G, et al. Distinct roles of IL-12 and IL-15 in human natural killer cell activation by dendritic cells from secondary lymphoid organs. Proc Natl Acad Sci U S A. 2004;101(47):16606-16611.

30. Daniel V, et al. Observational support for an immunoregulatory role of $\mathrm{CD} 3^{+} \mathrm{CD} 4^{+} \mathrm{CD} 25^{+} \mathrm{IFN}-\gamma^{+}$blood lymphocytes in kidney transplant recipients with good long-term graft outcome. Transpl Int. 2008;21(7):646-660.

31. Wang C, et al. CD8(+)NKT-like cells regulate the immune response by killing antigen-bearing DCs. Sci Rep. 2015;5:14124.

32. Sanchez-Fueyo A, Markmann JF. Immune exhaustion and transplantation. Am J Transplant. 2016;16(7):1953-1957.

33. Kumar S, et al. PD-1 expression on Foxp3 ${ }^{+}$Treg cells modulates CD8 ${ }^{+} \mathrm{T}$ cell function in prostatic tumor microenvironment. $J$ Immunol. 2017;198(1_suppl):155.11.

34. Sprangers B, Nair V, Launay-Vacher V, Riella LV, Jhaveri KD. Risk factors associated with post-kidney transplant malignancies: an article from the Cancer-Kidney International Network. Clin Kidney J. 2018;11(3):315-329.

35. Halloran PF, Famulski K, Reeve J. The molecular phenotypes of rejection in kidney transplant biopsies. Curr Opin Organ Transplant. 2015;20(3):359-367.

36. Parkes MD, Halloran PF, Hidalgo LG. Mechanistic sharing between NK cells in ABMR and effector T cells in TCMR. Am J Transplant. 2018;18(1):63-73.

37. Luo Q, et al. Comprehensive analysis of long non-coding RNA and mRNA expression profiles in rheumatoid arthritis. Exp Ther Med. 2017;14(6):5965-5973.

38. Wiebe C, et al. Class II HLA epitope matching-A strategy to minimize de novo donor-specific antibody development and improve outcomes. Am J Transplant. 2013;13(12):3114-3122.

39. Wiebe C, Nevins TE, Robiner WN, Thomas W, Matas AJ, Nickerson PW. The synergistic effect of class II HLA epitope-mismatch and nonadherence on acute rejection and graft survival. Am J Transplant. 2015;15(8):2197-2202.

40. Sapir-Pichhadze R, et al. HLA-DR and -DQ eplet mismatches and transplant glomerulopathy: a nested case-control study. Am $J$ Transplant. 2015;15(1):137-148.

41. Wu K, Budde K, Schmidt D, Neumayer HH, Rudolph B. Pathologic characteristics of early or late acute cellular rejection and outcome after kidney transplant. Exp Clin Transplant. 2014;12(4):314-322.

42. Jalalzadeh M, Mousavinasab N, Peyrovi S, Ghadiani MH. The impact of acute rejection in kidney transplantation on long-term allograft and patient outcome. Nephrourol Mon. 2015;7(1):e24439.

43. Lorenz EC, et al. Relationship between pre-transplant physical function and outcomes after kidney transplant. Clin Transplant. 
2017;31(5):10.1111/ctr.12952.

44. Loupy A, et al. Subclinical rejection phenotypes at 1 year post-transplant and outcome of kidney allografts. J Am Soc Nephrol. 2015;26(7):1721-1731.

45. Schinstock CA, et al. The value of protocol biopsies to identify patients with de novo donor-specific antibody at high risk for allograft loss. Am J Transplant. 2017;17(6):1574-1584.

46. Parajuli S, et al. Which is more nephrotoxic for kidney transplants: BK nephropathy or rejection? Clin Transplant. 2018;32(4):e13216.

47. Cadillo-Chávez R, et al. Assessing the risk of infection and rejection in Hispanic renal transplant recipients by means of an adenosine triphosphate release assay. Transplant Proc. 2006;38(3):918-920.

48. Solez K, et al. Banff 07 classification of renal allograft pathology: updates and future directions. Am J Transplant. 2008;8(4):753-760.

49. Haas M, et al. Banff 2013 meeting report: inclusion of c4d-negative antibody-mediated rejection and antibody-associated arterial lesions. Am J Transplant. 2014;14(2):272-283.

50. Ritchie ME, et al. limma powers differential expression analyses for RNA-sequencing and microarray studies. Nucleic Acids Res. $2015 ; 43(7):$ e47. 